\title{
Direct Observation of the Polarity Control Mechanism in Aluminum Nitride Grown on Sapphire by Aberration Corrected Scanning Transmission Electron Microscopy.
}

\author{
Lindsay Hussey $^{1}$, Isaac Bryan ${ }^{1}$, Ronny Kirste ${ }^{1}$, Wei Guo ${ }^{1}$, Zachary Bryan ${ }^{1}$, Seiji Mita ${ }^{2}$, Ramón Collazo ${ }^{1}$, \\ and Zlatko Sitar'. \\ 1. Department of Materials Science and Engineering, North Carolina State University, Raleigh, NC \\ 27695-7919, USA. \\ 2. HexaTech, Inc., 991 Aviation Pkwy, Suite 800, Morrisville, NC 27560, USA.
}

III-nitride materials are the foundation of modern light emitting diode (LED) technology [1-3]. The fabrication of LEDs based on III-nitride alloys has the potential for wavelength emission ranging from the near infrared to deep ultraviolet (DUV). Of the III-nitride materials, AlN has the largest band gap of $6.2 \mathrm{eV}$ [4], giving it the potential to be developed into DUV LEDs and lasers with applications that include compact sterilization tools for biomedical purposes. Additionally, the crystalline structure of IIInitrides provide the unique capability to exploit the chemical differences of the III-polar and N-polar crystal faces and develop improved electrical devices such as high electron mobility transistors and novel devices such as lateral polar junctions and periodically poled second harmonic generators [5-7]. In order to develop these AlN based devices, however, it is required that both Al-polar and N-polar AlN of high structural quality are synthesized in an efficient and reproducible manner.

The equilibrium crystal structure of all III-nitrides, including AlN, is that of wurtzite [4]. This crystal structure is non-centrosymmetric, and when grown along the c-direction, has an intrinsic polarization due to the electronegativity difference in nitrogen and aluminum atoms. These bond arrangements result in different chemical properties of the $+\mathrm{c}$ (Al-polar) and $-\mathrm{c}(\mathrm{N}$-polar) surfaces that effect affect material growth, surface quality, and impurity incorporation. Both Al-polar and N-polar AlN films can be grown on sapphire substrates by metalorganic chemical vapor deposition (MOCVD) with the appropriate sapphire surface treatment [8]. Growth of predominately N-polar AlN is observed on a sapphire surface that undergoes a low temperature anneal in an ammonia atmosphere, also called a nitridation step. Either the omission of nitridation or the implementation of a low temperature buffer layer before AIN deposition consistently results in an Al-polar film.

Verification of polarity is typically achieved indirectly by exploiting the chemical differences of the surface through the stark variation between Al-polar and N-polar AlN etch rates in a potassium hydroxide solution [9]. While useful as an indirect measurement tool, wet etching offers no information about the atomic structure of the interface between the sapphire substrate and the AlN film. Atomic resolution imaging of this interface is achieved by Scanning transmission electron microscopy (STEM). Additionally, recent advancements in the development of aberration corrected electron microscopes with a sub-Angstrom beam size has increased spatial resolution, allowing for the identification of polarity of structures with minimal variation in their atomic number, such as $\mathrm{Al}$ and $\mathrm{N}$ [10, 11]. In this paper, the origins at the atomic scale of how sapphire surface treatments result in N-polar and Al-polar AlN films are discussed. Aberration-Corrected STEM results suggest the mechanism of polarity control in AIN grown directly on nitrided sapphire to be the mimicry of N-polarity from N-polar AlN formed by nitridation of the sapphire surface. For the case of Al-polar films grown on AlN buffer layers, appropriate annealing conditions promote growth of Al-polar facets in the buffer layer and overgrowth of the N-polar AlN initially created on the sapphire surface. 


\section{References:}

[1] M. Asif Khan, K. Balakrishnan, and T. Katona, Nat. Photonics 277 (2008).

[2] Y. Taniyasu, M. Kasu and T. Makimoto. Nature 441325 (2006).

[3] M. Kneissl, T. Kolbe, C. Chua, V. Kueller, N. Lobo, J. Stellmach, A. Knauer, H. Rodriguez, S.

Einfeldt, Z. Yang, N. M. Johnson, and M. Weyers, Semicond. Sci. Technol. 26, 014036 (2011).

[4] S. Strite and H. Morkoc, J. Vac. Sci. Technol. B 10, 1237 (1992).

[5] S. Dasgupta, Nidhi, D.F. Brown, F. Wu, S. Keller, J.S. Speck, and U.K. Mishra, Appl. Phys. Lett.

96, 143504 (2010).

[6] R. Collazo, S. Mita, A. Aleksov, R. Schlesser and Z. Sitar, J. Cryst. Growth 287, 586 (2006).

[7] A. Chowdhury, H. M. Ng, M. Bhardwaj, and N. G. Weimann. Appl. Phys. Lett. 83, 1077 (2003).

[8] R. Kirste, S. Mita, L. Hussey, M.P. Hoffmann, W. Guo, I. Bryan, Z. Bryan, J. Tweedie, J. Xie, M.

Gerhold, R. Collazo, and Z. Sitar, Applied Physics Letters 102 (2013) p. 181913.

[9] D. Zhuang and J. H. Edgar, Materials Science and Engineering: R: Reports 48 (2005) p. 1.

[10] D. Muller, Nature Materials 8, (2009) p. 263.

[11] G. Van Tendeloo, S. Bals, S. Van Aert, J. Verbeeck, and D. Van Dyck, Advanced Materials 24 (2012), Adv. Mater. p. 5655.

[12] The authors acknowledge funding from the Graduate Women in Science (GWIS) Ethel Allen Fellowship.
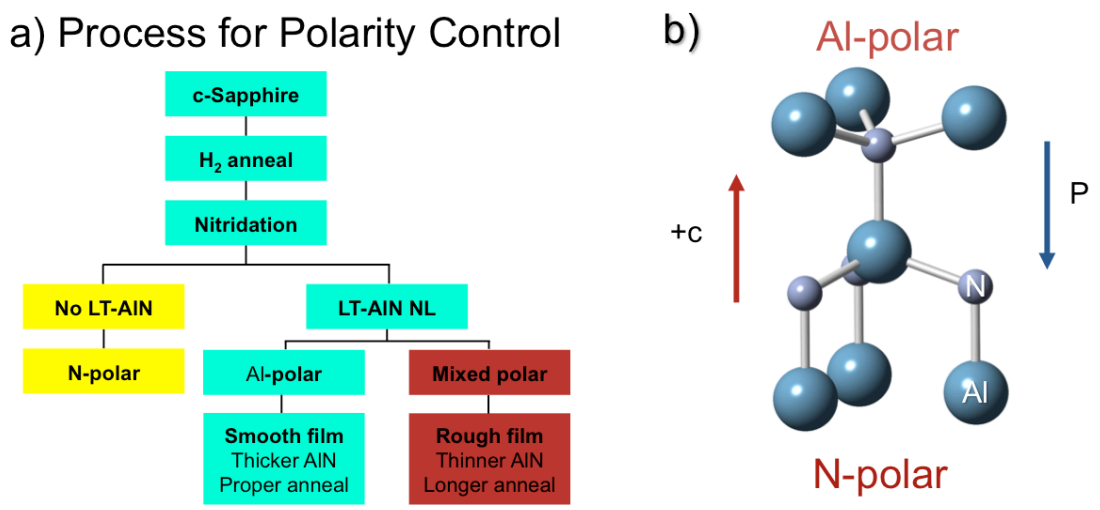

Figure 1. The a) process for polarity control of AlN grown on sapphire and b) partial wurtzite unit cell indicating Al- and N-polarity and the direction of polarization in AlN.

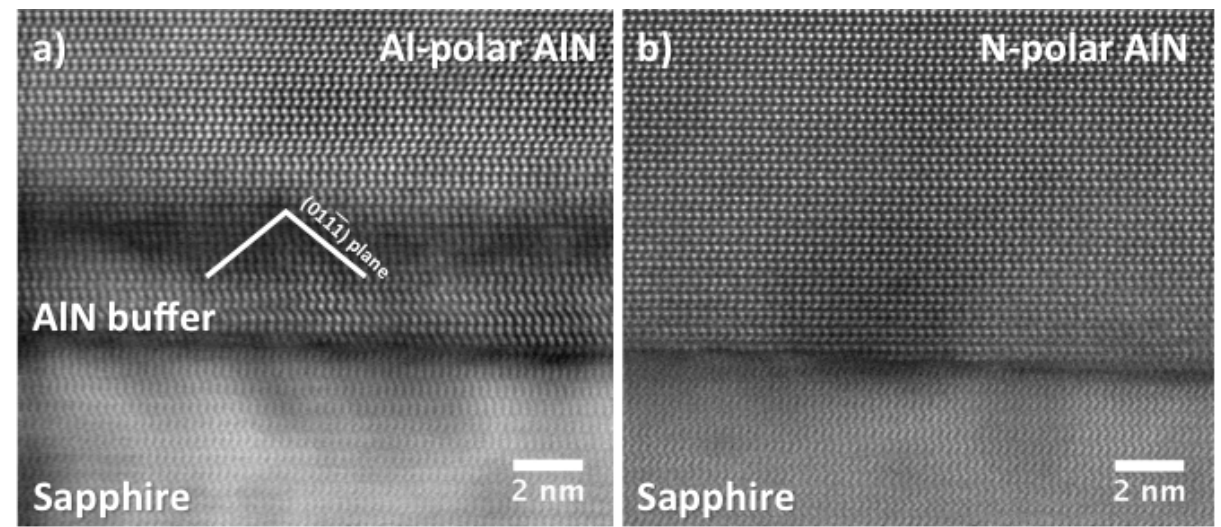

Figure 2. Aberration-corrected STEM images of a) an Al-polar and b) an N-polar AlN layers grown on sapphire substrates. Planes formed in the AlN buffer layer after annealing are indicated white lines in $b$ ). 